\section{Revista 2020 \\ Med Vol. 28(2)}

julio-diciembre
-
ISSN: 0121-5256 - e-ISSN: 1909-7700
Editorial Neogranadina

\title{
Hipotiroidismo en mujeres en la posmenopausia, prevalencia en el Eje Cafetero, Colombia, 2016-2019*
}

\author{
Franklin José Espitia De La Hoz ${ }^{\text {a }}$
}

\begin{abstract}
Resumen: el objetivo es determinar la prevalencia de hipotiroidismo en mujeres en la posmenopausia, en el Eje Cafetero. Materiales y métodos: estudio de corte transversal en 469 participantes. Se ingresaron mujeres mayores de 40 años, en la posmenopausia, que asistieron a la consulta externa para atención por patología ginecológica; entre julio de 2016 y junio de 2019, en tres clínicas privadas de carácter universitario, en el Eje Cafetero, Colombia. Se excluyeron mujeres con diagnóstico previo de hipotiroidismo o que se negaron a participar. Muestreo aleatorio simple. Variables medidas: sociodemográficas, clínicas y quirúrgicas. Se aplicó estadística descriptiva. Resultados: la edad media fue de 56,47 \pm 7,14 años. La media de los valores de la TSH en la población global fue de 3,71 \pm 1,94 $\mu \mathrm{UI} / \mathrm{mL}$, con tendencia al incremento a medida del aumento de la edad. La prevalencia de hipotiroidismo en mujeres en la posmenopausia fue del 48,61 \% ( $n=228 / 469)$ (IC95 \%: 37,83-54,15), en el Eje Cafetero; siendo más elevada en las obesas (54,41 \%; IC95 \%, 43,29-49,41\%) y en las mayores de 60 (52,35 \%; IC95 \%: 20,64-31,77). Se detectaron anticuerpos antitiroideos antiperoxidasa (Ac-TPO) en el 46,05 \% ( $n=105 / 228$ ) y los antitiroglobulina (TgAb) en el 21,05 \% ( $n=48 / 228$ ) de las mujeres hipotiroideas, evidenciándose un fenómeno autoinmune en el 3,26 \% ( $n=153 / 469)$ de la población total estudiada. El sobrepeso, el incremento de la edad y la presencia de anticuerpos antitiroideos aumentan significativamente la prevalencia de hipotiroidismo $(p<0,05)$. Conclusiones: el 48,61 \% de las mujeres del Eje Cafetero en la posmenopausia presentan hipotiroidismo.
\end{abstract}

Palabras clave: hipotiroidismo; mujeres; posmenopausia; prevalencia; receptores de tirotropina

Recibido: 5/03/2020. Aceptado: 03/07/2020

Disponible en línea: 04/06/2021

Cómo citar: Espitia De La Hoz FJ. Hipotiroidismo en mujeres en la posmenopausia, prevalencia en el Eje Cafetero, Colombia, 2016-2019. Rev. Med. [Internet]. 4 de junio de 2021 [citado 4 de junio de 2021];28(2):61-0. Disponible en: https://revistas.unimilitar.edu.co/index.php/rmed/article/view/4868

* Artículo de investigación.

a MD en Ginecología y Obstetricia, Hathor, Clínica Sexológica, Clínica del Café, Armenia (Colombia). Correo electrónico: espitiafranklin@hotmail.com ORCID: https://orcid.org/0000-0002-4581-9680 


\title{
Hypothyroidism in postmenopausal women, prevalence in the Coffee Region, Colombia, 2016-2019
}

\begin{abstract}
: the aim is to determine the prevalence of hypothyroidism in postmenopausal women in the Coffee Region. Materials and methods: cross-sectional study in 469 participants. Postmenopausal women over 40 years of age who sought outpatient care due to gynecological pathologies between July 2016 and June 2019 in three private university clinics in the Coffee Region, Colombia, were admitted. Women with a previous diagnosis of hypothyroidism or who refused to participate were excluded. Simple random sampling. Measured variables: sociodemographic, clinical, and surgical. Descriptive statistics were applied. Results: the mean age was $56.47 \pm 7.14$ years. The mean TSH values in the global population were $3.71 \pm 1.94 \mu \mathrm{IU} / \mathrm{mL}$, increasing with age. The prevalence of hypothyroidism in postmenopausal women was $48.61 \%$ ( $n=228 / 469)(95 \% \mathrm{CI}$ : 37.83-54.15) in the Coffee Region, being higher in obese women (54.41 \%; 95 \% CI, 43.29-49.41\%) and those over 60 (52.35\%; 95 \% CI: 20.64-31.77). Anti-thyroid peroxidase antibodies (TPOAb) were detected in $46.05 \%(n=105 / 228)$ and thyroglobulin antibodies (TgAb) in $21.05 \%(n=48 / 228)$ of hypothyroid women, showing an autoimmune phenomenon in $3.26 \%(n=153 / 469)$ of the total population studied. Overweight, older age and antithyroid antibodies increase the prevalence of hypothyroidism $(p<0.05)$. Conclusions: $48.61 \%$ of postmenopausal women from the Coffee Region have hypothyroidism.
\end{abstract}

Keywords: hypothyroidism; women; postmenopause; prevalence; thyrotropin receptors

\section{Hipotiroidismo em mulheres na pós-menopausa, prevalência no Eje Cafetero, Colômbia, 2016-2019}

Resumo: o objetivo é determinar a prevalência de hipotiroidismo em mulheres na pós-menopausa, no Eje Cafetero (Eixo Cafeeiro). Materiais e métodos: estudo de corte transversal em 469 participantes. Foram incluídas mulheres maiores de 40 anos, na pós-menopausa, que foram à consulta externa para o atendimento por patologia ginecológica; entre julho de 2016 e junho de 2019, em três hospitais particulares de caráter universitário, no Eje Cafetero, Colômbia. Foram excluídas mulheres com diagnóstico prévio de hipotiroidismo ou que negaram a participar. Amostragem aleatória simples. Variáveis medidas: sociodemográficas, clínicas e cirúrgicas. Foi aplicada estatística descritiva. Resultados: a idade média foi de $56,47 \pm 7,14$ anos. A média dos valores da TSH na população global foi de $3,71 \pm 1,94 \mu \mathrm{UI} / \mathrm{mL}$, com tendência à elevação à medida que a idade aumentasse. A prevalência de hipotiroidismo em mulheres na pós-menopausa foi de 48,61 \% ( $n=228 / 469)$ (IC95 \%: 37,83-54,15), no Eje Cafetero; sendo mais elevada nas obesas (54,41 \%; IC95 \%, 43,29-49,41 \%) e nas maiores de 60 (52,35\%; IC95 \%: 20,64-31,77). Foram detectados anticorpos antitiroideus antiperoxidase (AC-TPO) em 46,05\% ( $n=105 / 228)$ e os antitiroglobulina (TgAb) em $21,05 \%$ ( $n=48 / 228)$ das mulheres com hipotiroidismo, evidenciando-se um fenômeno autoimune em 3,26 \% ( $n=153 / 469)$ da população total estudada. O sobrepeso, o aumento da idade e a presença de anticorpos antitiroideus aumentam significativamente a prevalência de hipotiroidismo $(p<0,05)$. Conclusões: $48,61 \%$ das mulheres do Eje Cafetero na pós-menopausa apresentam hipotiroidismo.

Palavras-chave: hipotiroidismo; mulheres; pós-menopausa; prevalência; receptores de tirotropina 


\section{Introducción}

El hipotiroidismo es uno de los trastornos endocrinos más comunes, fácilmente identificable y fácilmente tratable, pero si no se diagnostica o no se trata, puede tener profundos efectos adversos. Se caracteriza por ser un síndrome con manifestaciones clínicas y bioquímicas de falla tiroidea y de déficit de disponibilidad de hormona tiroidea en los tejidos diana $(1,2)$.

En el hipotiroidismo primario, la hormona estimulante de la tiroides (TSH) se incrementa a más de $10 \mu \mathrm{UI} / \mathrm{mL}$, con una disminución simultánea de tiroxina (T4) libre (3). El hipotiroidismo subclínico se caracteriza por un aumento de la TSH $(5-10 \mu \mathrm{UI} /$ $\mathrm{mL}$ ) y T4 libre normal (4).

El hipotiroidismo es frecuente en todo el mundo. La deficiencia de yodo y la enfermedad autoinmune (tiroiditis de Hashimoto) representan la gran mayoría de los casos de hipotiroidismo primario (2).

En países con suficiente yodo, la prevalencia del hipotiroidismo varía entre $1 \%$ a $2 \%(5,6)$, aumentando a $7 \%$ en individuos de entre 85 y 89 años (7). En ausencia de rangos de referencia específicos de edad para TSH, se ha establecido la hipótesis de que existe la posibilidad de que una población que envejece tenga una mayor prevalencia de hipotiroidismo; de igual modo, el hipotiroidismo es aproximadamente diez veces más frecuente en mujeres que en hombres (3). Por su parte, el hipotiroidismo subclínico implica un diagnóstico de laboratorio, y su prevalencia se estima entre $12 \%$ y $18 \%(8,9)$.

El verdadero vínculo para desarrollar síntomas hipotiroideos es incierto, sin embargo, la progresión para presentar hipotiroidismo es de alrededor del $4 \%$ por año en presencia de Ac-TPO, y del $2 \%$ por año si no está presente (10).

La función tiroidea regula una amplia gama de parámetros metabólicos; puede afectar de forma significativa el metabolismo de las lipoproteínas, así como de algunos factores de riesgo de enfermedad cardiovascular (ECV), por lo tanto, influye en el riesgo general de ECV (11-13).

El hipotiroidismo también se acompaña de una mayor prevalencia del síndrome metabólico (14) y la relación cintura-cadera (15). La resistencia a la insulina aumenta en la insuficiencia tiroidea (16), mientras que se observa una disminución en los transportadores de glucosa GLUT4 (el que conduce a una reducción de la absorción de glucosa y promueve la resistencia a la insulina) $(16,17)$.

Los valores normales para la TSH, considerados en sangre, oscilan entre $0,5-4,5 \mu \mathrm{UI} / \mathrm{mL}$, con ligeras variaciones según el laboratorio. En las situaciones en las cuales se confirma una TSH elevada, con niveles normales de T4 libre, se hace el diagnóstico de hipotiroidismo subclínico. Se ha observado que valores mayores a $2 \mu \mathrm{UI} / \mathrm{mL}$, se consideran como de riesgo de enfermedad tiroidea si se tiene familiares con hipotiroidismo, anticuerpos antitiroideos, embarazo o tratamiento con fármacos que afectan la función tiroidea (amiodarona, ezetimiba, fenitoína, etc.) $(18,19)$.

A la fecha, en Latinoamérica la prevalencia de la disfunción tiroidea es variable, dependiendo de la edad, la raza y el sexo; observándose una mayor frecuencia en las mujeres que en los hombres (20), en los blancos y mulatos más que en los afrodescendientes (10\% de hipotiroidismo y $2,2 \%$ de hipertiroidismo). A su vez, la prevalencia del hipotiroidismo subclínico es $11,8 \%$ (21).

En Colombia son pocos los estudios que reportan la prevalencia del hipotiroidismo, encontrándose reportes del $18,5 \%$ en población de 35 y más años y del $38,75 \%$ en gestantes $(22,23)$; sin embargo, la mayoría de los estudios se limita a ciertas áreas o ciudades, y con una modesta población en la muestra. No se han realizado estudios regionales sobre la prevalencia del hipotiroidismo, y menos en la mujer posmenopáusica. Por eso, el objetivo del presente estudio consiste en determinar la prevalencia de hipotiroidismo en mujeres en la posmenopausia, en el Eje Cafetero.

\section{Materiales y métodos}

Diseño y población. Estudio observacional descriptivo de corte transversal, al que ingresaron mujeres mayores de 40 años, en la posmenopausia, reclutadas en la consulta externa de ginecología, entre el 1 de julio de 2016 y el 30 de junio de 2019, en tres clínicas privadas de carácter universitario y centros de referencia ubicadas en el Eje Cafetero, 
Colombia. Se excluyeron mujeres con diagnóstico previo de hipotiroidismo, consumidoras de medicamentos que alteran la TSH (amiodarona, dopamina, litio, prednisona y yoduro de potasio) o que se negaron a participar en el estudio. El tamaño de la muestra se calculó teniendo en cuenta el total de la población de mujeres del Eje Cafetero (según datos del Censo de 2018 realizado por el Dane), con una prevalencia esperada del $50 \%$, un margen de error del $5 \%$ y un índice de confianza del $95 \%$, resultando una muestra de 385 participantes. Se tomó un $20 \%$ más de la población estimada, para corregir el número de mujeres que no consintieran participar. En la selección de la muestra se hizo un muestreo aleatorio simple para la selección de las participantes, mediante números aleatorios generados por computadora.

Procedimiento. A todas las participantes se les diligenció un cuestionario que incluía los datos sociodemográficos, personales (patológicos, quirúrgicos, farmacológicos, salud sexual y reproductiva), familiares, hábitos. A cada mujer se le solicitó niveles de TSH, T4 libre y anticuerpos antitiroideos (antiperoxidasa / Ac-TPO y antitiroglobulina / $\mathrm{Tg} \mathrm{Ab}$ ) en suero, en todos los casos. Se reportó TsH en $\mu \mathrm{UI} / \mathrm{mL}$ y $\mathrm{T} 4$ libre en $\mathrm{ng} / \mathrm{dL}$.

La enfermedad tiroidea autoinmune, se definió como la coexistencia de anticuerpos antiperoxida$\mathrm{sa}($ Ac-TPO $)<30 \mathrm{UI} / \mathrm{mL}$ o antitiroglobulina (TgAb) $<35 \mathrm{UI} / \mathrm{mL}$, sin alteraciones en los parámetros séricos de las hormonas tiroideas o en las concentraciones de TsH. La enfermedad tiroidea autoinmune aislada, en mujeres eutiroideas, se definió con la coexistencia de Ac-TPO $>30 \mathrm{UI} / \mathrm{mL}$ o $\mathrm{TgAb}<35$ $\mathrm{UI} / \mathrm{mL}$ con concentraciones de TSH menores de 2,5 $\mu \mathrm{UI} / \mathrm{L}$ (24). Una vez hecha la toma de la muestra, la paciente era citada a la semana para seguimiento de los resultados. Se permitió un tiempo máximo de tres meses entre la valoración clínica y el seguimiento del reporte de las pruebas. Se utilizaron los puntos de corte recomendados por el U.S. Preventive Services Task Force para clasificar las pacientes según los resultados: a) Hipotiroidismo: TsH mayor a $4,5 \mu \mathrm{UI} / \mathrm{mL}$ y T4 libre menor de 0,7 ng/dL; b) Hipotiroidismo subclínico: TsH mayor o igual a $4,5 \mu \mathrm{UI} / \mathrm{mL}$ con T4 libre normal (0,7-1,9 $\mathrm{ng} / \mathrm{dL})(6,24,25)$. En los casos en que las mujeres presentaran niveles de TSH y T4 libre fuera de los rangos de normalidad, se citaban a una consulta individual para valoración por medicina interna, en la cual se encargaban del manejo y seguimiento. Una vez se hacía el diagnóstico de hipotiroidismo, se procedía a la búsqueda de la historia clínica de la paciente, en la cual se tomó la información clínica. Tres médicos generales verificaron los criterios de elegibilidad y extrajeron la información de los datos. La medición de la TsH y T4 libre se hizo mediante un método ultrasensible (quimioluminiscencia de tercera generación), detectando valores hasta de $0,0001 \mu \mathrm{UI} / \mathrm{mL}$, y leído por una bacterióloga con especialización en Química Clínica, y analizado por un médico especialista, integrante del equipo de investigación. En todas las clínicas participantes la técnica de procesamiento de la muestra fue quimioluminiscencia y se aceptó como nivel normal aquel valor dentro del rango de referencia informado por cada laboratorio.

Variables medidas: sociodemográficas (edad, etnia, nivel de escolaridad, estrato socioeconómico, estado civil, ocupación, afiliación al sistema general de seguridad social en salud, condición espiritual o religiosa, área de residencia); variables de salud sexual y reproductiva (edad de la menarquia, embarazos, abortos, paridad); variables de comportamiento sexual (orientación sexual, edad de la primera relación sexual); antecedentes personales, edad de la menopausia, uso de terapia hormonal de reemplazo; hábitos (tabaquismo, ingesta de alcohol, ingesta de café y sedentarismo). La información fue digitada en Microsoft 2011 Excel 14.0, aplicando validación de los datos en las celdas, a fin de evitar errores de digitación.

Análisis estadístico. La descripción de las variables cualitativas se resumió por medio de frecuencias absolutas y relativas para las variables cualitativas, las continuas mediante medias y desviaciones estándar o mediana y rangos, dependiendo de su distribución, debido a que no recibieron una distribución normal. Se describió la prevalencia general de hipotiroidismo (número de participantes con hipotiroidismo confirmado / total de participantes que ingresaron). Se calculó el intervalo de confianza del $95 \%$ para la prevalencia de hipotiroidismo. Para la prevalencia de 
hipotiroidismo subclínico (número de participantes con TsH elevada y T4 libre normal / total de participantes que ingresaron). Se hizo la descripción de los valores de TSH y T4 libre según los rangos de edad y se calculó la frecuencia de hipotiroidismo e hipotiroidismo subclínico por grupos etarios. Se hizo uso de las pruebas de $\chi^{2}$ de Pearson y Exacta de Fisher, se tomó como nivel de significación estadística un valor de $\mathrm{p}<0,05$. Los datos se analizaron con el programa SPSS versión 21.0.

Consideraciones éticas: el estudio se consideró sin riesgo, acorde con los principios éticos de la Declaración de Helsinki; recibió el aval del Comité de Ética e Investigación en Salud de Hathor, Clínica Sexológica.

\section{Resultados}

Se identificaron 1785 mujeres mayores de 40 años, en la posmenopausia, de estas se seleccionaron aleatoriamente 693 . Un total de $66(9,52 \%)$ fueron eliminadas por diagnóstico previo de hipotiroidismo, en 45 pacientes $(6,49 \%)$ no se pudo disponer de la historia clínica. De las 582 restantes, se excluyeron 75 (12,88 \%) por consumir medicamentos que alteran la TSH y $38(6,52 \%)$ se negaron a participar en el estudio, lo que dio como resultado un total de 469 participantes.

La edad promedio fue de 56,47 \pm 7,14 años. En su mayoría eran hispánicas, casadas o en unión libre, estrato medio, amas de casa, procedencia urbana $(86,99 \%)$, católicas $(85,71 \%)$, heterosexuales $(92,75 \%)$ y pertenecientes al régimen contributivo, en el sistema de seguridad social en Colombia $(88,91 \%)$. Las características sociodemográficas se describen en la Tabla 1. Según el IMC, en la población total el $69,72 \%(\mathrm{n}=327 / 469)$ tenía sobrepeso u obesidad (35,82 \% frente al 33,9 \%, respectivamente).

Tabla 1. Características socio-demográficas de las mujeres con hipotiroidismo, en la posmenopausia. Eje Cafetero, Colombia, 2016-2019, n = 469

\begin{tabular}{ll}
\hline Variables & $\mathrm{n}(\%)$ \\
\hline Edad (años), promedio (DE) & $56,47 \pm 7,14$ \\
\hline Edad (años) promedio (DE) de la pareja & $58,96 \pm 8,35$ \\
\hline Talla, mts & $1,64 \pm 13,79$ \\
\hline Peso, Kg & $67,86 \pm 6,53$ \\
\hline IMC, Kg/m 2 , promedio (DE) & $25,37 \pm 4,78$ \\
Raza & $267(56,92 \%)$ \\
\hline Hispánicas & $168(35,82 \%)$ \\
\hline Afro descendientes & $34(7,24 \%)$ \\
\hline Indígenas & \\
\hline Estado civil & $225(47,97 \%)$ \\
\hline Casada / Unión libre & $157(33,47 \%)$ \\
\hline Soltera & $54(11,51 \%)$ \\
\hline Divorciada & $33(7,03 \%)$ \\
\hline Viuda & \\
\hline Nivel de escolaridad & $137(15,55 \%)$ \\
\hline Primaria & $183(39,01 \%)$ \\
\hline Secundaria & $126(26,86 \%)$ \\
\hline Profesional & $73,56)$ \\
\hline
\end{tabular}




\begin{tabular}{ll}
\hline Ocupación & \\
\hline Amas de casa & $258(55,01 \%)$ \\
\hline Empleadas & $70(14,92 \%)$ \\
\hline Desempleadas & $96(20,46 \%)$ \\
\hline Pensionadas & $45(9,59 \%)$ \\
\hline Estrato socio-económico & \\
\hline Alto & $72(15,35 \%)$ \\
\hline Medio & $280(59,7 \%)$ \\
\hline Bajo & $117(24,94 \%)$ \\
\hline
\end{tabular}

Respecto a la salud sexual y reproductiva, el $78,67 \%$ de las mujeres había iniciado su vida sexual en el rango entre 15 y 19 años, el 17,27 \% después de los 19 años y el 8,95\% entre los 10 y 14 años. La mediana de compañeros sexuales reportó 4 (rango entre 0 y 9). La edad promedio al tener el primer hijo fue de 16,98 $\pm 2,15$ años. El 36,46 \% de las mujeres había estado embarazadas en 3 o más ocasiones, el 33,26 \% había dado a luz una sola vez y el 6,39 \% refirió haber abortado por lo menos en una ocasión. La paridad arrojó una mediana de 5 (rango entre 1 y 13 ).

En relación con los hábitos, el uso de cigarrillos fue del $14,71 \%$, el consumo de alcohol —de manera regular- se observó en el 66,52 \%, las que manifestaron practicar algún tipo de actividad física alcanzaron el 23,66 \%, mientras la ingesta de café se observó en el 95,94\%.

La edad de la menopausia reportó una media de 49,38 \pm 3,76 años. El 31,34 \% (IC95 \%: 26,43$37,59)$ de la población estaba en etapa de la posmenopausia temprana. El tiempo de menopausia reportó una media de 8,59 $\pm 2,57$ años. En cuanto a la terapia de reemplazo hormonal, el $41,15 \%$ no la utilizaba, el 19,82 \% se aplicaba terapia local vaginal, el 16,63\% usaba oral sistémica, el 5,75\% transdérmica y el 3,19 \% utilizaba la combinación de oral-local tópica vaginal.

La media de los valores de la TsH en la población global fue de 3,71 $\pm 1,94 \mu \mathrm{UI} / \mathrm{mL}$ y la T4 libre de $1,38 \mathrm{ng} / \mathrm{dL}$ (IC95 \%: 1,26-1,65).

La prevalencia global del hipotiroidismo fue del 48,61 \% ( $\mathrm{n}=228 / 469)$ (IC95 \%: 37,83-54,15); siendo más elevada en las mujeres obesas (54,41 \%; IC95 \%, 43,29-49,41\%) frente a las mujeres con normopeso (31,98 \%; IC95 \%: 29,19-34,23).
La prevalencia del hipotiroidismo estimada en la población de mujeres entre 40 y 49 años alcanzó el 8,33 \% (IC95 \%: 5,16-13,92); en las de 50 a 59 años fue del 19,17\% (IC95 \%: 16,95-23,97); y fue más elevada en las de 60 a 69 años (52,35\%; IC95 \%: 20,64-31,77).

La prevalencia global del hipotiroidismo subclínico alcanzó el 21,74 \% ( $\mathrm{n}=102 / 469)$ (IC95 \%: 18,45-22,95); se observó que la prevalencia alcanzó el 58,92 \% (IC95 \%: 47,31-61,59) en la población de mujeres con sobrepeso, el 68,35 \% (IC95 \%: 56,8573,92) en las obesas y el 48,51 \% (IC95 \%: 45,42$54,18)$ en las con normopeso.

La prevalencia de hipotiroidismo subclínico se calculó en el 6,42 \% (IC95 \%: 3,69-8,52) en las menores de 60 años y alcanzó el 19,01 \% (IC95 \%: 14,82-29,37) en el grupo de más de 60 años.

La media de la TSH en las mujeres sin hipotiroidismo fue de 2,95 $\mu \mathrm{UI} / \mathrm{mL}$ (IC95 \%: 1,08-3,87) y la T4 libre 1,74 ng/dL (IC95 \%: 1,11-1,92), mientras que en las mujeres con hipotiroidismo fue de 9,57 $\mu \mathrm{UI} / \mathrm{mL}$ (IC95 \%: 4,89-13,56) y la T4 libre de 0,57 ng/dL (IC95 \%: 0,18-0,87), observándose que existen diferencias estadísticamente significativas entre los valores de TSH y T4 libre de las mujeres con y sin hipotiroidismo $(\mathrm{p}=0,001)$. Se encontraron valores de TsH desde $<0,003 \mu \mathrm{UI} / \mathrm{mL}$ a 77,53 $\mu \mathrm{UI} / \mathrm{mL}$ y T4 libre desde $0,003 \mathrm{ng} / \mathrm{dL}$ a $2,74 \mathrm{ng} / \mathrm{dL}$.

Los anticuerpos antiperoxidasa (Ac-тPO) se detectaron en el 46,05 \% ( $n=105 / 228)$ y los anticuerpos antitiroglobulina ( $\mathrm{TgAb}$ ) en el 21,05\% ( $\mathrm{n}=48 / 228)$ de las mujeres hipotiroideas, evidenciándose un fenómeno autoinmune en el 3,26 \% ( $\mathrm{n}=153 / 469)$ de la población total estudiada. El 9,95\% de las mujeres eutiroideas presentó anticuerpos antitiroideos. El 2,48 \% de las mujeres 
estudiadas presentaron los dos anticuerpos, el 4,97 \% los Ac-тPо y ninguna presentó solamente $\operatorname{TgAb}$.

Se observó que la media de la TSH en las mujeres con enfermedad tiroidea autoinmune fue de 4,59 \pm $1,14 \mu \mathrm{UI} / \mathrm{L}$. De acuerdo con el tipo de anticuerpo positivo, se encontró que para las que tenían $\mathrm{Tg} \mathrm{Ab}$ positivos, la media de TsH fue de 5,37 $\pm 1,38 \mu \mathrm{UI} / \mathrm{L}$, con Ac-TPo positivos fue de 4,68 $\pm 1,56 \mu \mathrm{UI} / \mathrm{L}$, mientras que para ambos anticuerpos positivos la media de la TSH fue de $11,85 \pm 4,71 \mu \mathrm{UI} / \mathrm{L}$.

En las mayores de 60 años se encontró una disminución significativa de $\mathrm{TgAb}$ positivos, $\mathrm{y}$ un aumento importante de la TSH $(\mathrm{p}<0,05)$.

Las obesas presentaron un incremento significativo $(\mathrm{p}<0,05)$ de los niveles de la TsH con respecto a las con normopeso $(4,92 \pm 1,71 \mu \mathrm{UI} / \mathrm{mL}$ frente a $2,85 \pm 1,05 \mu \mathrm{UI} / \mathrm{mL}$ ).

Se observa que tanto el sobrepeso como la obesidad, así como el incremento de la edad y la presencia de anticuerpos antitiroideos, aumentan notablemente la prevalencia de hipotiroidismo $(\mathrm{p}<0,05)$.

\section{Discusión}

El hipotiroidismo es un trastorno endocrino de carácter asintomático o subclínico, en la mayoría de las personas, es más frecuente en mujeres y se incrementa con la edad (26). Según los estudios, su prevalencia varía entre 6-20\%, dependiendo de la edad, el sexo, la raza, la reposición de yodo de la población estudiada, así como el nivel de corte de la TsH empleado para definir su diagnóstico $(13,27,28)$. En la mayoría de los pacientes con tiroiditis autoinmune se encuentran presentes altas concentraciones de anticuerpos antitiroideos (predominando anticuerpos Ac-TPO y anticuerpos $\operatorname{Tg} \mathrm{Ab}$ ); también se detectan concentraciones elevadas de anticuerpos Ac-Tpo en el $11 \%$ de la población general (29). A su vez, en pacientes con hipotiroidismo subclínico, las mediciones de anticuerpos Ac-TPO ayudan a predecir la progresión a enfermedad manifiesta $(30,31)$.

En el presente estudio se descubrió que el hipotiroidismo es una forma común de disfunción tiroidea que afecta al 48,61 \% (IC95 \%: 37,83-54,15) de la población de mujeres en la posmenopausia, predominando en las mujeres mayores de 60 años, en quienes es seis veces mayor que en las menores de 50 años (52,35 \% frente al 8,33\%; p = 0,001). Se encontraron anticuerpos antitiroideos antiperoxidasa (Ac-TPO) en el 46,05 \% $(n=105 / 228)$ y antitiroglobulina (TgAb) en el 21,05 \% ( $\mathrm{n}=48 / 228)$ de las mujeres hipotiroideas, evidenciándose un fenómeno autoinmune en el 3,26 \% ( $\mathrm{n}=153 / 469)$ de la población total estudiada. El sobrepeso, el aumento de la edad y la presencia de anticuerpos antitiroideos, incrementan significativamente la prevalencia de hipotiroidismo $(\mathrm{p}<0,05)$ en la posmenopausia.

Los resultados de este estudio sugieren que en mujeres en la posmenopausia, en el Eje Cafetero, la prevalencia de hipotiroidismo es muy alta; se encontró que el 48,61 \% tenía hipotiroidismo no diagnosticado, mientras el hipotiroidismo subclínico se detectó en el 21,74 \%. Los resultados son similares a los informados por Acharya et al. (32), en el año 2011 en la India, quienes realizaron un estudio en 80 mujeres, y encontraron que el 42,5\% tenía hipotiroidismo manifiesto y el $57,5 \%$, hipotiroidismo subclínico, aunque fue un estudio en un grupo de mujeres en edad reproductiva. Por otro lado, Chandey et al. (33) concluyeron que el hipotiroidismo subclínico es más común, especialmente en las mujeres (26,6\%), y por lo general se presenta como manifestaciones vagas. Esto es de relevancia ya que el hipotiroidismo en los adultos mayores suele ser subdiagnosticado, en especial porque la sintomatología suele confundirse con efectos adversos de medicamentos, a la escasez de síntomas o por los cambios propios de la edad. De hecho, en el adulto mayor es usual una elevación normal o fisiológica de la TSH, con bajos niveles de triyodotironina libre (T3L) y T4 libre en límites normales bajos $(34,35)$.

Se sabe que aproximadamente el $95 \%$ de la tiroiditis autoinmune crónica tiene altos niveles de anticuerpos Ac-TPO, y aproximadamente el $60 \%$ de los pacientes tiene anticuerpos $\mathrm{TgAb}$, en particular en las formas atróficas (36); por lo tanto, cualquier valor de la TsH por encima de $4,6 \mu \mathrm{UI} / \mathrm{mL}$ debe considerarse anormal en referencia al diagnóstico de hipotiroidismo subclínico (37). 
Son varios los estudios que han demostrado que el hipotiroidismo subclínico se asocia con hiperlipidemia, síntomas neuromusculares, neuropsiquiátricos, disfunción miocárdica y disminución de la calidad de vida con progresión a hipotiroidismo manifiesto (37) y riesgo de enfermedad cardiovascular (38). Por eso se entiende que debido a las múltiples complicaciones sistémicas asociadas al hipotiroidismo, se hace necesario poner en marcha guías de manejo, a fin de facilitarle al clínico las formas de detectar las poblaciones de riesgo; puesto que, un diagnóstico temprano permite proponer un tratamiento eficaz y seguro, a fin de despejar las comorbilidades (39).

Se considera que el sobrepeso u obesidad, se relacionan con la alta prevalencia de hipotiroidismo detectado en esta población ya que el incremento del índice de masa corporal, como ha sido descrito por varios autores, puede estar asociado con el aumento sérico de la TSH (40-42) porque se ha demostrado la existente correlación entre los niveles de тsн y el porcentaje de grasa corporal (43).

Se necesitan ensayos clínicos aleatorizados para estudiar el efecto del tratamiento del hipotiroidismo en mujeres en la posmenopausia, lo cual también sería de particular interés en mujeres con hipotiroidismo subclínico o con anticuerpos antitiroideos positivos, para identificar sus efectos específicos a nivel metabólico en el envejecimiento femenino. Estos datos pueden resultar valiosos para establecer parámetros diagnósticos y terapéuticos con el propósito de mejorar la calidad de vida de estas mujeres. El estudio se podría ampliar a todo el país, con la inclusión de una mayor muestra, a fin de investigar las medidas para diagnosticar y controlar el hipotiroidismo, en un intento de reducir sus potenciales consecuencias negativas en la mujer en la posmenopausia.

La principal debilidad para destacar en este estudio es que resulta que por ser mujeres que consultaron a una institución de salud, podría haber un sesgo de selección, sin ser representativas de la población general. Entre las fortalezas sobresale el hecho de tener un muestreo aleatorio simple, ya que cada una de las mujeres tenía igualdad de oportunidades de ser seleccionada. Finalmente, se usaron técnicas de evaluación de las muestras siguiendo parámetros estandarizados, así como los criterios de hipotiroidismo primario y subclínico. Adicional a ello, el estudio se hizo en una zona colombiana donde ya se había detectado una significativa frecuencia de hipotiroidismo en el embarazo, además de coexistir una alta prevalencia de anticuerpos antitiroideos positivos, como lo describen Espitia et al. (23).

\section{Conclusiones}

La prevalencia de hipotiroidismo en mujeres en la posmenopausia es del 48,61 \% en el Eje Cafetero. Se evidencia un fenómeno autoinmune en el $3,26 \%$ de la población total estudiada. Las mujeres con sobrepeso, las mayores de 60 años y la presencia de anticuerpos antitiroideos incrementan la prevalencia.

\section{Agradecimientos}

El autor reconoce la valiosa contribución de un gran maestro y amigo, el doctor John Jairo Duque Ossman, en Armenia (Quindío).

\section{Financiación}

Esta investigación fue financiada con recursos propios del autor, haciendo turnos de obstetricia.

Conflicto de intereses: ninguno declarado.

\section{Referencias}

1. Jonklaas J, Bianco AC, Bauer AJ, Burman KD, Cappola AR, Celi FS, et al. Guidelines for the treatment of hypothyroidism: prepared by the American Thyroid Association task force on thyroid hormone replacement. Thyroid. 2014;24(12):1670-1751. DOI: https:// doi.org/10.1089/thy.2014.0028

2. Chaker L, Bianco AC, Jonklaas J, Peeters RP. Hypothyroidism. The Lancet. 2017;390(10101):1550-1562. DOI: https://doi.org/10.1016/S0140-6736(17)30703-1

3. Garber JR, Cobin RH, Gharib H, Hennessey JV, Klein I, Mechanick JI, et al. Clinical practice guidelines for hypothyroidism in adults: cosponsored by the American Association of Clinical Endocrinologists and the American Thyroid Association. Endocr Pract. 2012;18(6):988-1028. DoI: https://doi.org/10.4158/ EP12280.GL 
4. PearceSH, Brabant G, Duntas LH, MonzaniF, Peeters RP, Razvi S, et al. 2013 ETA guideline: management of subclinical hypothyroidism. Eur Thyroid J. 2013;2(4):21528. DoI: https://doi.org/10.1159/000356507

5. Vanderpump MP. The epidemiology of thyroid disease. Br Med Bull. 2011;99(1):39-51. DOI: https://doi. org/10.1093/bmb/ldr030

6. Parle JV, Franklyn JA, Cross KW, Jones SC, Sheppard MC. Prevalence and follow-up of abnormal thyrotrophin (TSH) concentrations in the elderly in the United Kingdom. Clin Endocrinol (Oxf). 1991;34(1):77-84. DOI: https://doi.org/10.1111/j.1365-2265.1991.tb01739.x

7. Gussekloo J, van Exel E, de Craen AJ, Meinders AE, Frölich M, Westendorp RG. Thyroid status, disability and cognitive function, and survival in old age. JAMA. 2004;292(21):2591-2599. DOI: https://doi.org/10.1001/ jama.292.21.2591

8. Almandoz JP, Gharib H. Hypothyroidism: etiology, diagnosis, and management. Med Clin North Am. 2012;96(2):203-221. DoI: https://doi.org/10.1016/j. mcna.2012.01.005

9. Baumgartner C, Blum MR, Rodondi N. Subclinical hypothyroidism: summary of evidence in 2014. Swiss Med Wkly. 2014;(144):w14058. DoI: https://doi. org/10.4414/smw.2014.14058

10. Vanderpump MP, Tunbridge WM, French JM, Appleton D, Bates D, Clark F, et al. The incidence of thyroid disorders in the community: a twenty-year follow-up of the Whickham Survey. Clin Endocrinol (Oxf). 1995; 43(1):55-68. DoI: https://doi. $\operatorname{org} / 10.1111 / j .1365-2265.1995 . t b 01894 . x$

11. Duntas LH. Thyroid disease and lipids. Thyroid. 2002;12(4):287-93. DoI: https://doi.org/10.1089/10507 250252949405

12. Friis T, Pedersen LR. Serum lipids in hyper- and hypothyroidism before and after treatment. Clin Chim Acta. 1987;162(2):155-63. DoI: https://doi.org/10.1016/00098981(87)90447-5

13. Canaris GJ, Manowitz NR, Mayor G, Ridgway EC. The Colorado thyroid disease prevalence study. Arch Intern Med. 2000;160(4):526-34. DoI: https://doi. org/10.1001/archinte.160.4.526

14. Raterman HG, van Eijk IC, Voskuyl AE, Peters MJ, Dijkmans BA, van Halm VP, et al. The metabolic syndrome is amplified in hypothyroid rheumatoid arthritis patients: a cross-sectional study. Ann Rheum Dis. 2010;69(1):39-42. DoI: https://doi.org/10.1136/ ard.2008.100776

15. Jung CH, Sung KC, Shin HS, Rhee EJ, Lee WY, Kim $\mathrm{BS}$, et al. Thyroid dysfunction and their relation to cardiovascular risk factors such as lipid profile, hs$\mathrm{CRP}$, and waist hip ratio in Korea. Korean J Intern Med. 2003;18(3):146-153. DoI: https://doi.org/10.3904/ kjim.2003.18.3.146

16. Lambadiari V, Boutati E, Nikzas D, Tountas N, Economopoulos T, Raptis SA, et al. Studies of insulin resistance in patients with clinical and subclinical hypothyroidism. Eur J Endocrinol. 2009;160(5):785790. DOI: https://doi.org/10.1530/EJE-08-0797

17. Shepherd PR, Kahn BB. Glucose transporters and insulin action-implications for insulin resistance and diabetes mellitus. N Engl J Med. 1999;341(4):248-257. DOI: https://doi.org/10.1056/NEJM199907223410406

18. Surks MI, Ortiz E, Daniels GH, Sawin CT, Col NF, Cobin $\mathrm{RH}$, et al. Subclinical thyroid disease: scientific review and guidelines for diagnosis and management. JAMA. 2004; 291(2):228-238. DoI: https://doi. org/10.1001/jama.291.2.228

19. Demers LM, Spencer CA. Laboratory medicine practice guidelines: laboratory support for the diagnosis and monitoring of thyroid disease. Clin Endocrinol (Oxf). 2003;58(2):138-140. DoI: https://doi.org/10.1046/ j.1365-2265.2003.01681.x

20. Guevara-Sánchez O, Holst-Schumacher I, Boza-Oreamuno S, Barrantes-Santamaría M, Chinchilla-Monge R, Alvarado-Ulate P. Disfunción tiroidea subclínica en población adulta costarricense. An Fac Med. 2015;76(4):333-338. DoI: https://doi.org/10.15381/anales.v76i4.11402

21. Fardella C. Epidemiología de los trastornos tiroideos en Chile. Boletín tiroideo. 2000;29.

22. Londoño ÁL, Gallego ML, Bayona A, Landázuri P. Prevalencia de hipotiroidismo y relación con niveles elevados de anticuerpos antiperoxidasa y yoduria en población de 35 y más años en Armenia. 2009-2010. Rev salud pública. 2011;13(6):998-1009. Dor: https:// doi.org/10.1590/S0124-00642011000600012

23. Espitia-De La Hoz FJ, Orozco-Santiago L. Prevalencia y caracterización clínica del hipotiroidismo, en gestantes del Eje Cafetero (Colombia), 2014-2017. Revista Colombiana de Endocrinología, Diabetes \& Metabolismo. 2019;6(4):260-267. Disponible en: http://revistaendocrino.org/index.php/rcedm/article/view/543/708

24. Hinojosa-Rodríguez KA, Martínez-Cruz N, Ortega-González C, López-Rioja MJ, Recio-López Y, Sánchez-González CM. Prevalencia de autoinmunidad tiroidea en mujeres subfértiles. Ginecol Obstet Mex. 2017;85(10):694-704. DOI: https://doi.org/10.24245/ gom.v85i10.1577 
25. Rugge JB, Bougatsos C, Chou R. Screening and treatment of thyroid dysfunction: an evidence review for the U.S. Preventive Services Task Force. Ann Intern Med. 2015;162(1):35-45. Dor: https://doi.org/10.7326/ M14-1456

26. Rallison ML, Dobyns BM, Meikle AW, Bishop M, Lyon JL, Stevens W. Natural history of thyroid abnormalities: prevalence, incidence, and regression of thyroid diseases in adolescents and young adults. Am J Med. 1991;91(4):363-370. DoI: https://doi.org/10.1016/0002-9343(91)90153-o

27. Tunbridge WM, Evered DC, Hall R, Appleton D, Brewis M, Clark F, et al. The spectrum of thyroid disease in a community: the Whickham survey. Clin Endocrinol (Oxf). 1977;7(6):481-493. DoI: https://doi. org/10.1111/j.1365-2265.1977.tb01340.x

28. Sichieri R, Baima J, Marante T, de Vasconcellos MT, Moura AS, Vaisman M. Low prevalence of hypothyroidism among black and Mulatto people in a population-based study of Brazilian women. Clin Endocrinol (Oxf). 2007;66(6):803-807. DoI: https://doi. org/10.1111/j.1365-2265.2007.02816.x

29. Hollowell JG, Staehling NW, Flanders WD, Hannon WH, Gunter EW, Spencer CA, et al. Serum TsH, T(4), and thyroid antibodies in the United States population (1988 to 1994): National Health and Nutrition Examination Survey (NHANES III). J Clin Endocrinol Metab. 2002;87(2):489-499. DoI: https://doi.org/10.1210/ jcem.87.2.8182

30. Effraimidis G, Strieder TG, Tijssen JG, Wiersinga WM. Natural history of the transition from euthyroidism to overt autoimmune hypo- or hyperthyroidism: a prospective study. Eur J Endocrinol. 2011;164(1):107113. DoI: https://doi.org/10.1530/EJE-10-0785

31. Walsh JP, Bremner AP, Feddema P, Leedman PJ, Brown SJ, O'Leary P. Thyrotropin and thyroid antibodies as predictors of hypothyroidism: a 13-year, longitudinal study of a community-based cohort using current immunoassay techniques. J Clin Endocrinol Metab. 2010;95(3):1095-1104. DoI: https://doi.org/10.1210/ jc. 2009-1977

32. Acharya N, Acharya S, Shukla S, Inamdar SA, Khatri M, Mahajan SN. Gonadotropin levels in hypothyroid women of reproductive age group. J Obstet Gynaecol India. 2011;61(5):550-553. DoI: https://doi.org/10.1007/ s13224-011-0079-7

33. Manish C, Ranjeet K, Gurinder M, Rahul M. Prevalence of hypothyroidism in adults by screening TsH: a study from North India. Int J Adv Med. 2016;3(1):44-46. Dor: https://doi.org/10.18203/2349-3933.ijam20160186
34. Papaleontiou M, Haymart MR. Approach to and treatment of thyroid disorders in the elderly. Med Clin North Am. 2012;96(2):297-310. Dor: https:/doi.org/10.1016/j.mcna.2012.01.013

35. LeFevre ML; U.S. Preventive Services Task Force. Screening for thyroid dysfunction: U.S. Preventive Services Task Force recommendation statement. Ann Intern Med. 2015;162(9):641-650. DoI: https://doi. org/10.7326/M15-0483

36. Yeh HC, Futterweit W, Gilbert P. Micronodulation: ultrasonographic sign of Hashimoto thyroiditis. J Ultrasound Med. 1996;15(12):813-819. DoI: https://doi. org/10.7863/jum.1996.15.12.813

37. Deshmukh V, Behl A, Iyer V, Joshi H, Dholye JP, Varthakavi PK. Prevalence, clinical and biochemical profile of subclinical hypothyroidism in normal population in Mumbai. Indian J Endocrinol Metab. 2013;17(3):454-459. DoI: https://doi.org/10.4103/2230 8210.111641

38. Bastenie PA. Hypothyroidism and Coronary heart disease. Acta Cardiol 1982;37(5):365-373. Dor: https:// doi.org/10.1111/j.1365-2044.1982.tb01139.x

39. Biondi B, Warftosky L. Treatment with thyroid hormone. Endocr Rev. 2014;35(3):433-512. Dor: https:// doi.org/10.1210/er.2013-1083

40. Fox CS, Pencina MJ, D’Agostino RB, Murabito JM, Seely EW, Pearce EN, et al. Relations of thyroid function to body weight: cross-sectional and longitudinal observations in a community-based sample. Arch Intern Med. 2008;168(6):587-592. DoI: https://doi. org/10.1001/archinte.168.6.587

41. Gopinath B, Liew G, Flood VM, Wang JJ, Kifley A, Leeder SR, et al. The association between weight gain and thyroid function in an older population. Arch Intern Med. 2008;168(20):2283-2284. DoI: https:/doi. org/10.1001/archinte.168.20.2283

42. Knudsen N, Laurberg P, Rasmussen LB, Bülow I, Perrild $\mathrm{H}$, Ovesen $\mathrm{L}$, et al. Small differences in thyroid function may be important for body mass index and the occurrence of obesity in the population. J Clin Endocrinol Metab. 2005;90(7):4019-4024. DoI: https:// doi.org/10.1210/jc.2004-2225

43. Ortega E, Pannacciulli N, Bogardus C, Krakoff J. Plasma concentrations of free triiodothyronine predict weight change in euthyroid persons. Am J Clin Nutr. 2007;85(2):440-445. DoI: https://doi.org/10.1093/ ajcn/85.2.440 